\title{
Research on the Knowledgeable System of Excavator Based on Ontology
}

\author{
Xinyu Wu
}

\author{
Automotive Engineering institute, Jiangxi University of Technology, Nanchang 330098, China
}

Keywords: Knowledgeable System; Ontology; Excavator

\begin{abstract}
The knowledgeable system of excavator takes the existing computer model as main content which involves a glittering array of knowledge, including engineering, design, services and computer information technology, etc., which is a complicated system project. This paper aims to analyze its connotation in the engineering field. Artificial intelligence researchers directly introduce the term of ontology to demonstrate their research on its ontology in knowledge engineering. As a result, in understating ontology, artificial intelligence and information system tend to adopt logic theory or logical semantics, which is deviated from the term in engineering field. This paper firstly introduces the basic knowledge of ontology and then tries to construct the knowledge structure of excavator according to abstract thought. Finally, based on the above research results, this paper aims to adopt the basic theory as well as the method of thinking to describe the knowledgeable system of excavator so as to construct it based on XML description.
\end{abstract}

\section{Introduction}

In recent years, researches, development and application in terms of ontology gradually emerge in the computer science community and industrial circles. The demonstration of ontology-based excavator is able to formally describe the conceptual definition, relation among various concepts, approaches to operate and transfer, relation between the definition of functional model and functional model based on the knowledgeable system of excavator. Therefore, this paper mainly starts the research from the following three aspects: Firstly, it is about the knowledge engineering and XML language with ontology as the core which is the basic toll to realize the advancing of this system; secondly, it is the research on the existing operational system of excavator which can help to establish abstracted simulation and can be used in ontology language description; thirdly, it adopts 01L language to describe the knowledge ontology of excavator and then uses XMLSchema description to describe the excavator knowledge based on ontology. Excavator, as an important type in terms of heavy-duty machinery, its original heavy and clumsy types have change into refined and skilful types, which is obvious in the running system and operation system (which are core technological parts of excavator). At present, along with the advancement of technology, developing countries develop the technology quickly. What's more, the improvement cycle in terms of stability of locomotive performance and function enhancement is shorter. Moreover, the model type is also changing. 


\section{Approaches}

The knowledgeable system of excavator takes the existing computer model as main content which involves a glittering array of knowledge, including engineering, design, services, and computer information technology, etc., which is a complicated system project. In the course of developing excavator's knowledgeable system, it takes the excavator knowledge as the center to explore the establishment approaches and tools of exploring excavator ontology in order to realize the mutual understanding between computer system and working staffs and computer system. Based on this, it aims to achieve intelligent human-computer interaction, interoperation among various computer systems as well as the knowledge reusing in computer system.

Guarin takes the level of detail and field dependence as two basic dimensions to divide the ontology, in which, the level of detail can be beckoned as reference and low level detail aims to share the ontology.

Based on the field dependence, ontology can be divided into four types, such as top ontology, field ontology, task ontology and application ontology:

Firstly, it is the top ontology. It describes the relation between common ideas which are always abstract terms. What's more, it is not related with concrete application. Other types can be deemed as the examples of this one which does not need formalization.

Secondly, it is the field ontology which describes the relation between the concept of specific fields (for example, electronics, medicines, machines, and transportation fields), which is concrete with a formalized type and easily to be shared.

Thirdly, it is the task ontology which describes the concept relation of special fields and tasks.

Fourthly, application ontology: it describes the relation between special circles and various tasks which contains all of the knowledge (generally, it is the combination between field ontology and approach ontology) and the ontology realized in this paper belongs to this type.

Ontology is able to provide a common vocabularies and ideas for the communication between two human beings, two organizations, even human beings and organizations to reach a consensus.

Standard model: Concerning large software system, people with different backgrounds have to have a common understanding of system and its objectives to establish a standardized model, otherwise it is impossible to communicate.

Relation network: Nowadays, ontology can be used to set up a network of relationships, figure out the contents of the connection and their relationships so that searching information and operation can be realized based on the network.

Consistency and clarity: the role ontology playing in the communication aims to provide a clear definition for the term of the software system as well as the identical understanding of the same thing, which is definite.

Integrate concepts of different users: Ontology is able to integrate the different ideas of different users to form a comprehensive and holistic view.

One way is to design a translator to convert two different languages, which is shown in Fig. 1. Each double arrows line represents a translation between the two languages, and in this way, On (z) translators are needed.

Another method is to adopt ontology as Interlingua to support translation which is shown in Fig. 1. Concerning $\mathrm{N}$ languages, it is able to reduce the translators to On (), because only one translator is needed to convert the language. 


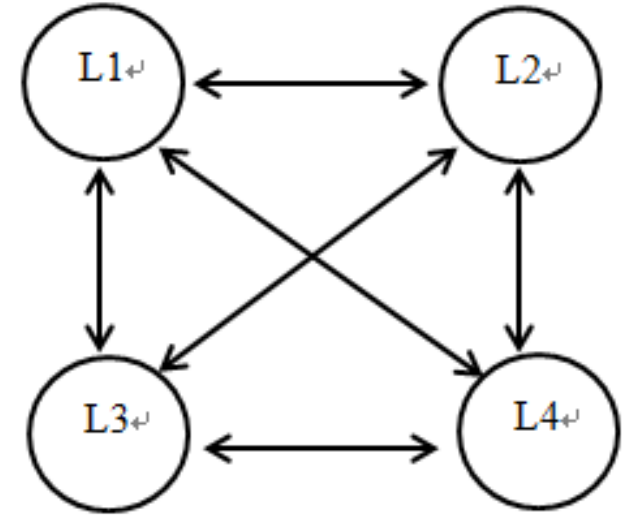

(a)

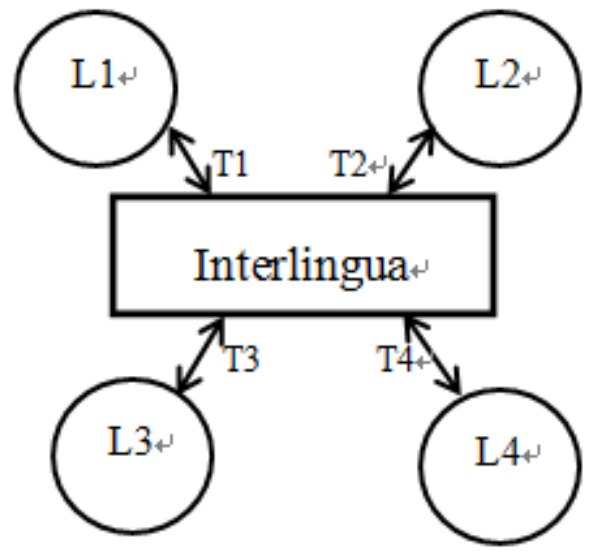

(b)

Fig. 1 two different conversion approaches

The application of ontology in information retrieval engine adopts ontology to describe the information to retrieve knowledge repositories (text, images, and Web pages), aiming to improve the efficiency and accuracy. Other items which need technical support include overview, search engines, automatic annotation of ontology, automatic classific ation, natural language processing, knowledge representation, large-scale knowledge base management and inference system etc. Different systems focus on different aspects and ontology plays different roles in these systems. In general, there are two types:

Firstly, the ontology is taken as the basis for structured information and knowledge. On the one hand, ontology, as an ideal framework, can help users to understand and organize the knowledge base. On the other hand, ontology also provides vocabularies ftor the system metadata to annotate data and profiles.

Secondly, ontology can be regarded as formalized representation. The visual ontology-driven interface helps users to form and search the process and even improve the accuracy of information based on the provided inference mechanism without user interaction.

XML (eXtensibleMarkupLanguage, Extensible Markup Language) is a standard to demonstrate and exchange internet data issued by W3C (WorldwidewbeConsortium) in February 1988. This language is a simplified subset of SGML (Standard Generalized Markup Language). XML combines the rich features of SGML and HTML accessibility in Web applications, which is a kind of new content-oriented technology with more structures and semantic meanings, good scalability, self-describing ability. What's more, it is easy to master and use. It makes the originally cluttered information which is unable to extend become flexible and reputable. As a result, the information change among different applications becomes unprecedentedly convenient. To conclude, it has been widely supported and it has showed great vitality.

Early artificial intelligence's researches on ontology models are in lack of general application rules. XML has been developed since 1996 when it has been beyond the meaning of page markup language and becomes the formalized description of object as well as the description language for distributed data switching. Similarly, ontology models which are established based on normalizing makes the definition and sharing of ontology mode become simple. Firstly, the concept of ontology model comes from Frame-based approach, and the tree type document structure of XML is able to 
successfully realize the mutual corresponding of them. What's more, XML document structure has abundance and expansibility as well as internal relevance which meets ontology's requirement of describing knowledge. There is another thing that XML has strictly regulated processing procedures, so it is easy to use and operate. Therefore, the ontology model that this paper analyzes is the knowledge representation and processing model related to technology based on XML.

The basic information of excavators is composed of descriptive data to describe the heavy machinery and to elaborate the inherent properties of the machine. The information includes manufacturer, classification (the most popular crawler hydraulic excavator is focused on), model number, date of production, date of sale, the number and so on.

The configured system of typical crawler hydraulic excavator system, engine system, hydraulic pressure system (taking JCM923B as an example) are made up of seven systems: walking support system, devices, assistive systems and electrical systems.

Hydraulic transmission takes liquid as working medium to transmit energy and control the mode of transmission. The hydraulic pressure system adopts hydraulic pumps to convert the mechanical energy of prime mover to fluid pressure energy in order to impart energy. It passes through a variety of control valves and piping, asking for the help of hydraulic actuator (cylinders and motors) to change liquid pressure energy to mechanical energy in order to drive machinery and realize straight reciprocating motion as well as rotational motion.

A complete hydraulic system consists of five parts: power components, actuators, control components, auxiliary components and hydraulic oil.

Power symbol is a hydraulic pump in the hydraulic pressure system, and its function is to convert the mechanical energy to liquid pressure to provide power for the entire hydraulic system. The structure of pump has cogwheel pump, sliding-vane pump and plunger pump. By the way, hydraulic excavator often adopts plunger pump

Actuator refers to hydraulic cylinders and hydraulic motors, and its function is to convert liquid pressure energy into mechanical energy in order to drive loading to complete straight reciprocating motion as well as rotational motion.

Control component refers to a glittering array of hydraulic valve and its role is to control and regulate the liquid pressure flow and direction. According to the different control functions, hydraulic valves can be divided into the following types:

1. Pressure control valve: overflow valve, pressure relief valve, sequence valve, pressure relays, etc.

2. Flow Control Valve: throttle valve, speed-regulating valve, flow distributing and collecting valve and so on.

3. Directional control valve: single valve, pilot operated check valve, shuttle valve and circuit change valve.

4. Other valves: hydraulic excavator also uses proportional solenoid, etc.

Auxiliary components include fuel tanks, oil filters, tubing and fittings, seals, pressure gauge, fuel level sensors, temperature sensors, etc.

Based on what is discussed above, XMLSChema can help to realize the excavator ontology based on $01 \mathrm{~L}$ language description which includes the following procedures: Firstly, list the concept system of ontology in detail (including class and slot restrictions); then change the slot to eomplexType in XMLSchmea (if the slot has domain and range, domain and range can be taken as elements and given definition). Besides, the eomplexType element of XMLSchemea (for example 
the subclass) should be changed. Finally, an element in each class can be established and be slotted to complete the XMLSchema description.

\section{Conclusions}

This paper firstly introduces the ontology and the carries out abstract analysis on the excavator's knowledge system; finally, based on the above research results, this paper applies both the theoretical thoughts and mode of thinking to the knowledge system description to form the ontology-based knowledge system of excavator with XML description method.

To conclude, the contents include the following points:

1. The research is on basic theory, including basic research on ontology and research on XML, XMLSchmea. It introduces the origins, concepts, functions and construction steps of ontology. What's more, it studies the related ontology tools in order to finally realize this construction of excavator ontology.

2. It analyzes the knowledgeable system of excavator and studies the ontology operation system to establish abstract construction for ontology language description.

3. It researches the description method based on XML to describe the knowledgeable system of excavator. Firstly, it analyzes the parts related to the ontology description based on XML language, introducing the description method of OLI to realize the knowled ge and the ontology description of excavator which is the core work in this paper.

4. This paper realizes the OIL description ontology with XMLSchema. After researching the relevant functions of XMLSchema, it finds out approaches. Based on this, it carries out concrete implementation, which is the final result.

\section{Acknowledgements}

This work was financially supported by the key subject building project (vehicle engineering) of Jiangxi University of Technology.

\section{Reference}

[1] Panas A, Pantouvakis J P. Evaluating research methodology in construction productivity studies[J]. The Built \& Human Environment Review, 2011, 3(1).

[2] Zhang Z, Li X, Liu Y, et al. Distributed Resource Environment: A Cloud-Based Design Knowledge Service Paradigm[M]//Cloud-Based Design and Manufacturing (CBDM). Springer International Publishing, 2014: 63-87.

[3] Johnston R A. Land and society: the Bronze Age cairnfields and field systems of Britain[J]. 2001.

[4] Cox J W, Hassard J. Ties to the past in organization research: A comparative analysis of retrospective methods[J]. Organization, 2007, 14(4): 475-497.

[5] Robinson D W. Drawing Upon the Past: Temporal Ontology and Mythological Ideology in South-central Californian Rock Art[J]. Cambridge Archaeological Journal, 2013, 23(03): 373-394.

[6] Cox J W, Hassard J. In their introduction to critical management research, Alvesson and Deetz[J]. SAGE Directions in Organization Studies, 2009, 14(4): 145. 
[7] Wu D. Cloud-Based Design and Manufacturing: A Network Perspective[J]. 2014.

[8] Mizoguchi K. The reproduction of archaeological discourse: the case of Japan[J]. Journal of European Archaeology, 1997, 5(2): 149-165.

[9] Larsen S F. Remembering and the archaeology metaphor[J]. Metaphor and Symbol, 1987, 2(3): 187-199.

[10] Van Belle J, Philips J, Ali O, et al. A service-oriented approach for holonic manufacturing control and beyond[M]//Service Orientation in Holonic and Multi-Agent Manufacturing Control. Springer Berlin Heidelberg, 2012: 1-20. 